\title{
Codebook Design and Performance Analysis of Quantized Beamforming under Perfect and Imperfect Channel State Information
}

\author{
Samuel T. Valduga, André L. F. de Almeida, Renato Machado, Andrei P. Legg, Murilo B. Loiola \\ and Dimas I. Alves
}

\begin{abstract}
This paper presents a codebook design to multiple transmit and multiple receive antenna system with quantized feedback channel. The codebook design is based on trigonometrical expressions that give a quasi-orthogonal structure to the codes. The codebook is designed to a specific number of transmit antennas and can easily be adapted to different number of feedback bits. An instantaneous signalto-noise ratio analysis is performed which is used to optimize the codebooks. An upper bound on the bit error rate (BER) performance for the proposed scheme is provided as well. We also investigate the system performance under more realistic conditions, and for this purpose we consider a time-varying and spatially-correlated channel model. We compare the BER performance of the proposed scheme with other closed-loop codebook-based schemes. Results show that the proposed scheme outperforms other good schemes in terms of array gain.
\end{abstract}

Index Terms-MIMO, beamforming, quantized feedback, BER and channel estimation.

\section{INTRODUCTION}

Quantized transmit beamforming is an interesting solution for multiple input multiple output (MIMO) systems since the directional signal can improve the signal-to-noise ratio (SNR) at the receiver enhancing the channel capacity [1] and reducing the bit error probability of the system [2]. For a quantized feedback system, a performance enhancement will depend on the system design and, obviously, on the available feedback information. When analog full channel state information (CSI) is available to the transmitter, optimal beamforming is the best strategy to be considered [3].

Deployment of multiple antennas is normally associated with high costs since multiple antennas usually require

Samuel T. Valduga and André L. F. de Almeida are with the Federal University of Ceará (UFC), Campus do Pici, s/n, CP 600560455-970, Fortaleza, CE, Brazil (e-mail: \{samueltv, andre\}@gtel.ufc.br).

Renato Machado is with the Aeronautics Institute of Technology (ITA), São José dos Campos - SP, 12228-900, Brazil (e-mail: renatomachado@ieee.org)

Andrei P. Legg is with the Federal University of Santa Maria (UFSM),

Santa Maria, RS, 97105-900, Brazil (e-mail: andrei.legg@gmail.com).

Murilo B. Loiola is with the Federal University of ABC (UFABC), Santo André, SP, 09210-170, Brazil (e-mail: murilo.loiola@ufabc.edu.br).

Dimas I. Alves is with the Federal University of PAMPA (UNIPAMPA), Alegrete, RS, 97546-550, Brazil (e-mail: dimas.alves@unipampa.edu.br).

The authors would like to thank the Brazilian agency CNPq, the Brazilian Army, the Swedish-Brazilian Research and Innovation Center (CISB), and Saab AB for the financial support.

Parts of this paper were presented at the IEEE Wireless Communications and Networking Conference (IEEE WCNC 2014), Istanbul, Turkey, 6-9 April 2014.

Digital Object Identifier: 10.14209/jcis.2017.16 an equal number of radio frequency (RF) chains (transmit amplifiers, modulators, etc.), which are expensive and powerconsuming. To circumvent this problem, a subset of the available antennas can be selected, thereby reducing the number of RF chains. The amount of feedback required in this case is much smaller. Such an approach is called transmit antenna selection (TAS). The most well-known TAS scheme was proposed by Gore and Paulraj [4] and later an extension of this idea was presented in [5]. Another interesting way of using the feedback information is by considering codebookbased (CBB) designs, where the feedback information is used to select a codeword from a finite codebook [2].

Along the last years, several works have investigated the use of quantized feedback in multiple-antenna systems [6]-[14]. Codebook design techniques based on Grassmann and Grassmannian-Stiefel manifold were proposed in [6], [7], where the main idea was to optimize the use of the quantized space of the eigendirections for MIMO channels. In the same direction, other codebook designs could be mentioned, such as random vector quantization (RVQ), discrete Fourier transform (DFT), and Lloyd codebooks, where a comparative analysis among them can be found in [8].

A class of extended orthogonal space-time block codes (EO-STBCs) for MIMO channels considering four transmit antennas over quasi-static flat fading channels was proposed in [9]. The spatial transmission rate those codes is unitary, since two symbols are transmitted over two periods of symbol. Based on the feedback information, a preprocessing is performed where the phases of the transmit symbols are rotated in a way to maximize the instantaneous SNR. As a result, the EO-STBC outperformed previous rate-one closedloop STBCs with four transmit antennas. Zhu et al. presented an analysis on symbol error rate (SER) of maximum ratio transmission, transmit antenna selection, and codebook-based beamforming in correlated Rayleigh fading channels [10]. They have derived an upper bound on the average SER of those schemes for a quantized CSI. Choi et al. proposed another phase-feedback-assisted scheme with four transmit antennas which uses a preprocessor to combine two Alamouti codes aiming at the Frobenius norm maximization [11]. They showed that full diversity is achieved by the combination made through preprocessing. Moreover, the feedback information was used to increase the coding gain. Herein, we refer to this scheme as Alamouti-code based scheme (ACBS). 
Transmit beamforming codebooks with separate amplitude and phase quantization was presented in [12]. The aim is either to maximize transmission rate (egoistic beamforming) or to minimize co-channel interference (altruistic beamforming). In [13] a MIMO scheme based on quasi-orthogonal spacetime block code (QOSTBC) for four transmit antennas was proposed. The idea is to eliminate the cross interference terms using the transmit antenna shuffling. Thus, an optimum antenna shuffling pattern is selected to improve the transmit diversity with limited feedback information over four time slots (modulation instants). However, this scheme was already outperformed by [9] in terms of bit error rate (BER) performance. Furthermore, some approaches considering precoding codebook based on the well-know DFT matrices are found in [14], where the main contribution is the lowcomplexity of the codebooks since the pre-processing is based on simple trigonometrical expressions.

In this context, we have proposed in [15] a codebook design for four-transmit-antenna quantized beamforming system. The codebook design is based on trigonometrical expressions that give a quasi-orthogonal structure to the codes. We also carried out an instantaneous SNR analysis which was taken into account to optimize the codebooks. Therein, an upper bound on the average BER performance is provided, which is used to show that the proposed scheme also achieves full diversity.

In this paper, the work presented in [15] is extended to a more general solution, where the proposed scheme is adjustable for any number of transmit antennas that is a multiple of four. Moreover, we assess the system performance under more realistic conditions, where a timevarying and spatially-correlated channel model [16] is adopted, and two channel estimation methods are considered [3], [17]. Additionally, the BER performance of the proposed scheme is compared with other closed-loop codebook-based schemes with four transmit antennas and unitary spatial transmission rate, which allows a more comprehensive performance evaluation.

The rest of the paper is organized as follows. Section II presents the system model. Section III addresses the proposed codebook design and the SNR analysis. In Section IV, an upper bound on the BER performance is derived. In Section V, we present the channel model considering Doppler and spatial correlation. In Section VI, we extend the proposal to MIMO for $M_{T}=4 N$ transmit antennas, and results are presented and discussed. Finally, Section VII presents some concluding remarks.

Throughout this paper, normal letters represent scalar quantities, boldface lowercase letters indicate vectors, and boldface uppercase letters indicate matrices. The superscripts 'T', ' $*$ ', '( $)^{H}$, represent the transpose, the complex conjugate and the conjugate transpose operation, $|\cdot|^{2}$ denotes the modulus squared of a complex number, $\Re\{\cdot\}$ the real part of a complex number and ' $\odot$ ' is the Hadamard product.

\section{SYSTEM MODEL}

Consider a MIMO system with $M_{T}$ antennas at the transmitter and $M_{R}$ antennas at the receiver. The channel is assumed to be quasi-static, flat Rayleigh fading, being constant over a frame and varying randomly from one frame to the next. The transmission model consists of a preprocessing based on trigonometrical expressions, which is given by

$$
\mathbf{Y}=\sqrt{\rho \mathbf{H P}}+\mathbf{N}
$$

where $\mathbf{Y}$ is the $M_{R} \times \tau$ matrix of received signals and $\mathbf{P}$ is the $M_{T} \times \tau$ matrix of processed transmitted signals with unit average energy. Let $\mathcal{C N}(0, \mathbf{R})$ represent the joint probability density function (p.d.f.) of a zero-mean circularly symmetric complex normal random vector with covariance matrix $\mathbf{R}$. Then, $\mathbf{N}$ is the $M_{R} \times \tau$ matrix $\mathcal{C N}\left(\mathbf{0}, \mathbf{I}_{\tau M_{R}}\right)$ representing the joint p.d.f. of the independent and identically distributed (i.i.d.) additive Gaussian noise samples with unit variance, $\mathbf{H}$ is the $M_{R} \times M_{T}$ MIMO channel characterized by the p.d.f. $\mathcal{C N}\left(\mathbf{0}, \mathbf{I}_{M_{R} M_{T}}\right)$, and $\rho=E_{b} / N_{0}$, which is the SNR associated to each receive antenna. $E_{b}$ is the average bit energy and $N_{0}$ is the noise variance.

We assume the information bits are mapped into a baseband unitary average energy constellation, such as PSK or QAM, giving rise to $Q$ data symbols $\left\{s_{q}\right\}, q=1, \ldots, Q$, to be transmitted over $\tau$ symbol periods. In this paper, we consider only schemes with unitary spatial transmission rate, i.e., $R=\frac{Q}{\tau} \log _{2}(M)=\log _{2}(M)$. In order to simplify the analytical derivations, we also assume that the channel coefficients are perfectly estimated at the receiver and that there is a reliable feedback channel through which $b$ bits can be sent to the transmitter. In Section V we consider more realistic assumptions in order to assess the system performance under more realistic scenarios.

\section{PRoposed SCHEME}

We start this section by considering the proposed transmission scheme for four transmit antennas as was presented in [15].

Assume that only one information symbol, $s$, is transmitted per transmit period. The symbol $s$ is preprocessed by a complex transmission codeword $\mathbf{x}$ :

$$
\mathbf{p}=s \mathbf{x}
$$

where

$$
\begin{aligned}
\mathbf{x} & =\left[\left(\left[\begin{array}{cc}
\cos (\theta) / \sqrt{2} & 0 \\
0 & \sin (\theta) / \sqrt{2}
\end{array}\right] \otimes \mathbf{I}_{2}\right) \mathbf{v}_{\varphi}\right] \\
& =\frac{1}{\sqrt{2}}\left[\begin{array}{c}
\cos (\theta) e^{j \varphi_{0}} \\
\cos (\theta) e^{j \varphi_{1}} \\
\sin (\theta) e^{j \varphi_{0}} \\
\sin (\theta) e^{j \varphi_{2}}
\end{array}\right]
\end{aligned}
$$

$\otimes$ is the Kronecker product, $\mathbf{I}_{2}$ is the 2-by-2 identity matrix, the factor $\frac{1}{\sqrt{2}}$ is used to normalize the transmit power and $\mathbf{v}_{\varphi}=\left[\begin{array}{llll}e^{j \varphi_{0}} & e^{j \varphi_{1}} & e^{j \varphi_{0}} & e^{j \varphi_{2}}\end{array}\right]^{\mathrm{T}}$. For simplicity of analysis, we assume that $\varphi_{0}=0$ and henceforth we follow with this assumption. It is worth mentioning that all of the analyses presented in this paper apply to any other $\varphi_{0} \neq 0$.

Before each transmission takes place, the receiver feeds back $b$ bits to the transmitter. The information is used by the preprocessor to choose the appropriate codeword, i.e., the 
one that maximizes the instantaneous $\mathrm{SNR}, \mathbf{x}_{\max } \in \mathbf{X}=$ $\left[\mathbf{x}_{1}, \mathbf{x}_{2}, \ldots, \mathbf{x}_{N}\right]$, where $N=2^{b}$. As one can see, the codebook is a function of $\varphi_{1}, \varphi_{2}$ and $\theta$, which are the variables that must be optimized to maximize the system performance.

Next, we present the receiver for $M_{R}$ receive antennas, the SNR analysis, used to optimize $\varphi_{1}, \varphi_{2}$ and $\theta$ in a unquantized space, and then the codebook design for $N=2^{b}$.

\section{A. Receiver for $M_{R}$ receive antennas}

From (1) and (2), the received signal $\mathbf{y}$ for $M_{R}$ receive antennas can be rewritten as

$$
\begin{aligned}
\mathbf{y} & =\mathbf{H p}+\mathbf{n}, \\
& =s \mathbf{H x}+\mathbf{n} \\
& =\overline{\mathbf{h}}+\mathbf{n}
\end{aligned}
$$

where

$$
\overline{\mathbf{h}}=s\left[h_{p 1} \ldots h_{p M_{R}}\right]^{\mathrm{T}}
$$

in which

$$
\begin{gathered}
h_{p j}=\cos (\theta) h_{1, j}+\cos (\theta) e^{\varphi_{1}} h_{2, j} \\
+\sin (\theta) h_{3, j}+\sin (\theta) e^{\varphi_{2}} h_{4, j}
\end{gathered}
$$

and

$$
\mathbf{H}=\left[\begin{array}{cccc}
h_{1,1} & h_{2,1} & h_{3,1} & h_{4,1} \\
\vdots & \vdots & \vdots & \vdots \\
h_{1, M_{R}} & h_{2, M_{R}} & h_{3, M_{R}} & h_{4, M_{R}}
\end{array}\right]
$$

where $\mathbf{n}$ is the $1 \times M_{R}(\tau=1)$ additive white Gaussian noise vector, and $h_{i, j}$ denotes path gain from the $i$-th transmit antenna to the $j$-th receive antenna.

Considering the received signal in (4) and employing the maximal ratio combining (MRC) technique, the following processing produces the desired inputs to the maximumlikelihood detector:

$$
\begin{aligned}
\tilde{s}= & \overline{\mathbf{h}}^{H} \mathbf{y} \\
= & s\left[\left(\sum_{j=1}^{M_{R}}\left(\left|h_{1, j}\right|^{2}+\left|h_{2, j}\right|^{2}\right)+\beta_{c}\right) \cos (\theta)^{2}\right. \\
& +\left(\sum_{j=1}^{M_{R}}\left(\left|h_{3, j}\right|^{2}+\left|h_{4, j}\right|^{2}\right)+\beta_{s}\right) \sin (\theta)^{2} \\
& \left.+\beta_{c s} \cos (\theta) \sin (\theta)\right] \sqrt{\rho} / 2+\sum_{j=1}^{M_{R}} h_{p j}^{*} \eta_{j}, \\
\beta_{c}= & \sum_{j=1}^{M_{R}} \beta_{c j}, \quad \beta_{s}=\sum_{j=1}^{M_{R}} \beta_{s j}, \quad \beta_{c s}=\sum_{j=1}^{M_{R}} \beta_{c s j}, \\
\beta_{c j}= & 2 \Re\left\{h_{1, j} h_{2, j}^{*} e^{-j \varphi_{1}}\right\}, \\
\beta_{s j}= & 2 \Re\left\{h_{3, j} h_{4, j}^{*} e^{-j \varphi_{2}}\right\}, \\
\beta_{c s j}= & 2 \Re\left\{\left(h_{1, j}+h_{2, j} e^{j \varphi_{1}}\right)\left(h_{3, j}+h_{4, j} e^{j \varphi_{2}}\right)^{*}\right\} .
\end{aligned}
$$

respectively, where $\kappa=g_{s}-g_{c}$. The third step is necessary to make sure that the solution is a maximum and not a minimum. The solving (10) under the conditions of $\gamma^{\prime}=0$ and $\gamma^{\prime \prime}<0$, we obtain the following optimal $\theta$

$$
\theta_{o p t}=\arctan \left(\frac{\kappa+\sqrt{\kappa^{2}+2 \beta_{c s}^{2}}}{\beta_{c s}}\right) .
$$

We decide to omit the term $\rho$ in (10), since this condition does not cause any alteration in the final result.

\section{Codebook Design}

In this section we present how to perform the proposed scheme based on quantized feedback. We assume two uniform finite sets: $\varphi_{1 q}$ and $\varphi_{2 q} \in[0, \pi]$ and $\theta_{q} \in[-\pi / 2, \pi / 2]$. Since the receiver needs to feed back the state information about two phases $\left(\varphi_{1 q}\right.$ and $\left.\varphi_{2 q}\right)$ and one angle $\left(\theta_{q}\right)$, the 
minimum quantization is, therefore, $b=3$ bits. The receiver computes and compares $2^{b}$ instantaneous SNR and send $b$ bits through the feedback channel to inform which code from the codebook must be considered for transmission. This process is repeated before a new transmission takes place. The system configuration for $b=3$ is presented in Table I.

TABLE I

SYSTEM CONFIGURATION FOR $b=3$. THIS IS THE MINIMUM QUANTIZATION THAT YIELDS FULL DIVERSITY FOR THE PROPOSED SCHEME

\begin{tabular}{c|c|c|c|c|c|c|c|c|c|c}
\hline \multicolumn{3}{c|}{$\gamma$} & \multicolumn{3}{c|}{ Parameters } & \multicolumn{3}{c|}{ Angle-Phases } & \multicolumn{3}{c}{ Feedback bits } \\
\hline \hline$\beta_{c}$ & $\beta_{s}$ & $\beta_{c s}$ & $\kappa$ & $\theta_{q}$ & $\varphi_{1 q}$ & $\varphi_{2 q}$ & $b_{0}$ & $b_{1}$ & $b_{2}$ \\
\hline$>0$ & $>0$ & $>0$ & $\sharp$ & $\frac{\pi}{4}$ & 0 & 0 & 0 & 0 & 0 \\
\hline$>0$ & $<0$ & $>0$ & $\sharp$ & $\frac{\pi}{4}$ & 0 & $\pi$ & 0 & 0 & 1 \\
\hline$<0$ & $>0$ & $>0$ & $\sharp$ & $\frac{\pi}{4}$ & $\pi$ & 0 & 0 & 1 & 0 \\
\hline$<0$ & $<0$ & $>0$ & $\sharp$ & $\frac{\pi}{4}$ & $\pi$ & $\pi$ & 0 & 1 & 1 \\
\hline$>0$ & $>0$ & $<0$ & $\sharp$ & $-\frac{\pi}{4}$ & 0 & 0 & 1 & 0 & 0 \\
\hline$>0$ & $<0$ & $<0$ & $\sharp$ & $-\frac{\pi}{4}$ & 0 & $\pi$ & 1 & 0 & 1 \\
\hline$<0$ & $>0$ & $<0$ & $\sharp$ & $-\frac{\pi}{4}$ & $\pi$ & 0 & 1 & 1 & 0 \\
\hline$<0$ & $<0$ & $<0$ & $\sharp$ & $-\frac{\pi}{4}$ & $\pi$ & $\pi$ & 1 & 1 & 1 \\
\hline
\end{tabular}

TABLE II

SYSTEM CONFIGURATION FOR $b=4$.

\begin{tabular}{c|c|c|c|c|c|l|l|l|l|l}
\hline \multicolumn{3}{c|}{$\gamma$ Parameters } & \multicolumn{3}{c|}{ Angle-Phases } & \multicolumn{3}{c}{ Feedback bits } \\
\hline \hline$\beta_{c}$ & $\beta_{s}$ & $\beta_{c s}$ & $\kappa$ & $\theta_{q}$ & $\varphi_{1 q}$ & $\varphi_{2 q}$ & $b_{0}$ & $b_{1}$ & $b_{2}$ & $b_{3}$ \\
\hline$>0$ & $>0$ & $>0$ & $>0$ & $\frac{3 \pi}{8}$ & 0 & 0 & 0 & 0 & 0 & 0 \\
\hline$>0$ & $<0$ & $>0$ & $>0$ & $\frac{3 \pi}{8}$ & 0 & $\pi$ & 0 & 0 & 0 & 1 \\
\hline$<0$ & $>0$ & $>0$ & $>0$ & $\frac{3 \pi}{8}$ & $\pi$ & 0 & 0 & 0 & 1 & 0 \\
\hline$<0$ & $<0$ & $>0$ & $>0$ & $\frac{3 \pi}{8}$ & $\pi$ & $\pi$ & 0 & 0 & 1 & 1 \\
\hline$>0$ & $>0$ & $>0$ & $<0$ & $\frac{\pi}{8}$ & 0 & 0 & 0 & 1 & 0 & 0 \\
\hline$>0$ & $<0$ & $>0$ & $<0$ & $\frac{\pi}{8}$ & 0 & $\pi$ & 0 & 1 & 0 & 1 \\
\hline$<0$ & $>0$ & $>0$ & $<0$ & $\frac{\pi}{8}$ & $\pi$ & 0 & 0 & 1 & 1 & 0 \\
\hline$<0$ & $<0$ & $>0$ & $<0$ & $\frac{\pi}{8}$ & $\pi$ & $\pi$ & 0 & 1 & 1 & 1 \\
\hline$>0$ & $>0$ & $<0$ & $>0$ & $\frac{-\pi}{8}$ & 0 & 0 & 1 & 0 & 0 & 0 \\
\hline$>0$ & $<0$ & $<0$ & $>0$ & $\frac{-\pi}{8}$ & 0 & $\pi$ & 1 & 0 & 0 & 1 \\
\hline$<0$ & $>0$ & $<0$ & $>0$ & $\frac{-\pi}{8}$ & $\pi$ & 0 & 1 & 0 & 1 & 0 \\
\hline$<0$ & $<0$ & $<0$ & $>0$ & $\frac{-\pi}{8}$ & $\pi$ & $\pi$ & 1 & 0 & 1 & 1 \\
\hline$>0$ & $>0$ & $<0$ & $<0$ & $\frac{-3 \pi}{8}$ & 0 & 0 & 1 & 1 & 0 & 0 \\
\hline$>0$ & $<0$ & $<0$ & $<0$ & $\frac{-3 \pi}{8}$ & 0 & $\pi$ & 1 & 1 & 0 & 1 \\
\hline$<0$ & $>0$ & $<0$ & $<0$ & $\frac{-3 \pi}{8}$ & $\pi$ & 0 & 1 & 1 & 1 & 0 \\
\hline$<0$ & $<0$ & $<0$ & $<0$ & $\frac{-3 \pi}{8}$ & $\pi$ & $\pi$ & 1 & 1 & 1 & 1 \\
\hline
\end{tabular}

The symbol $\sharp$ means “don’t care state”. Each row of Table I defines a codeword $\mathbf{x}\left(\varphi_{1}, \varphi_{2}, \theta\right)$. Phases $\varphi_{1}$ and $\varphi_{2}$ are used to keep $\beta_{c}$ and $\beta_{s}$ as non negative real numbers. As the number of feedback bits is increased, $\beta_{c}$ and $\beta_{s}$ will converge to the ideal (unquantized) solution, as presented in Section III-B. The angle $\theta$ is used to maximize the sum of the terms that compose the instantaneous SNR. The codebook is given by

$$
\mathbf{X}_{8}^{4}=\left[\begin{array}{cccc}
\cos (\pi / 4) & \cos (\pi / 4) & \sin (\pi / 4) & \sin (\pi / 4) \\
\cos (\pi / 4) & \cos (\pi / 4) & \sin (\pi / 4) & \sin (\pi / 4) e^{j \pi} \\
\cos (\pi / 4) & \cos (\pi / 4) e^{j \pi} & \sin (\pi / 4) & \sin (\pi / 4) \\
\cos (\pi / 4) & \cos (\pi / 4) e^{j \pi} & \sin (\pi / 4) & \sin (\pi / 4) e^{j \pi} \\
\cos (-\pi / 4) & \cos (-\pi / 4) & \sin (-\pi / 4) & \sin (-\pi / 4) \\
\cos (-\pi / 4) & \cos (-\pi / 4) & \sin (-\pi / 4) & \sin (-\pi / 4) e^{j \pi} \\
\cos (-\pi / 4) & \cos (-\pi / 4) e^{j \pi} & \sin (-\pi / 4) & \sin (-\pi / 4) \\
\cos (-\pi / 4) & \cos (-\pi / 4) e^{j \pi} & \sin (-\pi / 4) & \sin (-\pi / 4) e^{j \pi}
\end{array}\right]^{\mathrm{T}}
$$

Table II defines the system configuration for $b=4$ and the respective codebook is given by $\mathbf{X}_{16}^{4}$. The larger the number of feedback bits, the better the codebook performance. To improve the BER performance we can increase the quantization of $\theta$. On the other hand, by increasing the quantization of $\varphi$ there is no considerable improvement in performance.

$$
\mathbf{X}_{16}^{4}=\left[\begin{array}{cccc}
\cos (3 \pi / 8) & \cos (3 \pi / 8) & \sin (3 \pi / 8) & \sin (3 \pi / 8) \\
\cos (3 \pi / 8) & \cos (3 \pi / 8) & \sin (3 \pi / 8) & \sin (3 \pi / 8) e^{j \pi} \\
\cos (3 \pi / 8) & \cos (3 \pi / 8) e^{j \pi} & \sin (3 \pi / 8) & \sin (3 \pi / 8) \\
\cos (3 \pi / 8) & \cos (3 \pi / 8) e^{j \pi} & \sin (3 \pi / 8) & \sin (3 \pi / 8) e^{j \pi} \\
\cos (-3 \pi / 8) & \cos (-3 \pi / 8) & \sin (-3 \pi / 8) & \sin (-3 \pi / 8) \\
\cos (-3 \pi / 8) & \cos (-3 \pi / 8) & \sin (-3 \pi / 8) & \sin (-3 \pi / 8) e^{j \pi} \\
\cos (-3 \pi / 8) & \cos (-3 \pi / 8) e^{j \pi} & \sin (-3 \pi / 8) & \sin (-3 \pi / 8) \\
\cos (-3 \pi / 8) & \cos (-3 \pi / 8) e^{j \pi} & \sin (-3 \pi / 8) & \sin (-3 \pi / 8) e^{j \pi} \\
\cos (\pi / 8) & \cos (\pi / 8) & \sin (\pi / 8) & \sin (\pi / 8) \\
\cos (\pi / 8) & \cos (\pi / 8) & \sin (\pi / 8) & \sin (\pi / 8) e^{j \pi} \\
\cos (\pi / 8) & \cos (\pi / 8) e^{j \pi} & \sin (\pi / 8) & \sin (\pi / 8) \\
\cos (\pi / 8) & \cos (\pi / 8) e^{j \pi} & \sin (\pi / 8) & \sin (\pi / 8) e^{j \pi} \\
\cos (-\pi / 8) & \cos (-\pi / 8) & \sin (-\pi / 8) & \sin (-\pi / 8) \\
\cos (-\pi / 8) & \cos (-\pi / 8) & \sin (-\pi / 8) & \sin (-\pi / 8) e^{j \pi} \\
\cos (-\pi / 8) & \cos (-\pi / 8) e^{j \pi} & \sin (-\pi / 8) & \sin (-\pi / 8) \\
\cos (-\pi / 8) & \cos (-\pi / 8) e^{j \pi} & \sin (-\pi / 8) & \sin (-\pi / 8) e^{j \pi}
\end{array}\right]^{-\pi / 8}
$$

The feedback information, $b_{0}, \ldots, b_{n-1}$, is sent according to the rules defined for each system configuration. The codebook design for higher quantization.

\section{BER ANALYSIS}

In this section, we present a BER analysis for the proposed codebook-based beamforming. The analysis is performed for PSK constellations. The BER of $M$-ary PSK constellation conditioned on the instantaneous SNR $\gamma$ can be given by [18]:

$$
\operatorname{BER}(\gamma)=\frac{1}{\pi} \int_{0}^{\frac{(M-1) \pi}{M}} \exp \left(-\frac{C_{m} \gamma}{\sin ^{2}(\theta)}\right) d \theta
$$

where $M$ is the constellation size and $C_{m}=\sin ^{2}(\pi / M)$. Taking the expectation over (12), the average BER can be written as

$$
\overline{B E R}(\gamma)=\mathbb{E}_{h}\{B E R(\gamma)\}
$$

where $\mathbb{E}_{h}$ is the expectation operator with respect to $h$.

By assuming that the transmitter uses feedback information for choosing a codeword $\mathbf{x}$, which belongs to a certain finite codebook $\mathbf{X}$, such that the instantaneous SNR is maximized, we can rewritten (13) as

$$
\overline{B E R}(\rho)=\frac{1}{\pi} \int_{0}^{\frac{(M-1) \pi}{M}} \mathbb{M}\left(-\frac{C_{m} \rho}{\sin ^{2}(\theta)} ; \mathbf{X}\right) d \theta
$$

where $\mathbb{M}\left(-\frac{C_{m} \rho_{x}}{\sin ^{2}(\theta)} ; \mathbf{X}\right)$ denotes the value of the function

$$
\begin{aligned}
\mathbb{M}(t ; \mathbf{X}) & \triangleq \mathbb{E}\left\{\exp \left(-t \max _{\mathbf{x} \in \mathbf{X}}\left|\mathbf{h}_{p}\right|^{2}\right)\right\}, t \geq 0 \\
& =\mathbb{E}\left\{\exp \left(-t \max _{\mathbf{x} \in \mathbf{X}}|\mathbf{H} \mathbf{x}|^{2}\right)\right\}, t \geq 0
\end{aligned}
$$

As in [10], we consider the following approximation of (15):

$$
\beta(\mathbf{X})=\lim _{t \rightarrow \infty} t^{M_{T} M_{R}} \mathbb{M}(t ; \mathbf{X})
$$

in which the values assumed by $\beta(t, \mathbf{X})$ depend on the set of codewords in $\mathbf{X}$. 
This asymptotic approximation is fundamental for our analysis since an exact expression is very hard to obtain. However, if the codebook is well designed, and that is the case here, then the codewords can be approximated by 'spherical caps' on the surface of hypersphere, which simplifies considerably the BER analysis [19].

Based on the results presented in [19] and [10] we have the following approximation of (16)

$$
\begin{array}{r}
\beta(\mathbf{X})=C_{1}\left(M_{T} M_{R}-1\right) !\left(M_{T}-1\right) N \\
\times \sum_{n=0}^{M_{T}-2}\left(\begin{array}{c}
M_{T}-2 \\
n
\end{array}\right) \frac{(-1)^{n}\left(C_{2}^{-\left(M_{T} M_{R}-1-n\right)}-1\right)}{M_{T} M_{R}-1-n}
\end{array}
$$

where

$$
C_{1}=M_{T} M_{R} \prod_{n=1}^{\min \left(M_{T} M_{R}\right)} \frac{\left(\min \left(M_{T} M_{R}\right)-n\right) !}{\left(M_{T} M_{R}-1\right) !}
$$

and

$$
C_{2}=1-N^{\frac{-1}{M_{T}-1}}
$$

\section{A. An upper bound on the BER performance}

In this section, we continue with the analysis of $\beta(\mathbf{X})$, whose result will be used in (14) culminating in an upper bound on the average BER. The normalized channel is defined by $\tilde{\mathbf{h}_{p}} \triangleq \frac{\mathbf{h}_{p}}{\left|\mathbf{h}_{p}\right|}$ where $\left|\mathbf{h}_{p}\right|$ is independent of its direction. Furthermore, since $\mathbf{h}_{p}$ is Gaussian, $\left|\mathbf{h}_{p}\right|^{2}$ is a chisquare random variable with $2 M_{T} M_{R}$ degree of freedom and has the following moment generating function (MGF) $\mathbb{E}\left\{\exp \left(-s\left|\mathbf{h}_{p}\right|^{2}\right)\right\}=(1+s)^{-M_{T} M_{R}}$.

Applying the MGF to (15), we obtain

$$
\begin{aligned}
\mathbb{M}(t ; \mathbf{X}) & =\mathbb{E}_{\tilde{\mathbf{h}_{p}}}\left\{\mathbb{E}_{\left|\tilde{\mathbf{h}_{p}}\right|^{2}}\left\{\exp \left[-\left(t \Omega\left|\tilde{\mathbf{h}_{p}}\right|^{2}\right)\left|\mathbf{h}_{p}\right|^{2}\right]\right\}\right\} \\
& =\mathbb{E}_{\tilde{\mathbf{h}_{p}}}\left\{\left((1+t \Omega)\left|\tilde{\mathbf{h}_{p}}\right|^{2}\right)^{-M_{T} M_{R}}\right\}
\end{aligned}
$$

where the subscript on the expectation operator indicates the random variable is being averaged over, and $\Omega$ is defined as

$$
\Omega \triangleq\left(\max _{\mathbf{x} \in \mathbf{X}}\left|\tilde{\mathbf{h}_{p}}\right|^{2}\right)^{-M_{T} M_{R}} .
$$

Hence, we can rewrite (16) as

$$
\begin{aligned}
\beta(\mathbf{X}) & =\lim _{t \rightarrow \infty} t^{M_{T} M_{R}} \mathbb{M}(t ; \mathbf{X}) \\
& =\lim _{t \rightarrow \infty} t^{M_{T} M_{R}} \mathbb{E}_{\tilde{\mathbf{h}_{p}}}\left\{\left(1+\Omega^{\frac{-1}{M_{T} M_{R}}}\right)^{-M_{T} M_{R}}\right\} \\
& =\mathbb{E}_{\tilde{\mathbf{h}_{p}}}\{\Omega\} .
\end{aligned}
$$

For $t>0$, it is possible to affirm that $\left(1+t \Omega^{\frac{-1}{M_{T} M_{R}}}\right)-M_{T} M_{R}$ is concave with respect to $\Omega$. Thus, applying Jensen's inequality to (18), yielding:

$$
\begin{aligned}
\mathbb{M}(t ; \mathbf{X}) & \leq\left\{1+t\left(\mathbb{E}_{\tilde{\mathbf{h}_{p}}}\left\{\Omega^{\frac{-1}{M_{T} M_{R}}}\right\}\right)^{-M_{T} M_{R}}\right\} \\
& =\left\{1+t\left(\beta(\mathbf{X})^{\frac{-1}{M_{T} M_{R}}}\right)^{-M_{T} M_{R}}\right\} .
\end{aligned}
$$

Substituting (21) into (14), an upper bound on the average BER is obtained

$$
\begin{aligned}
& \overline{B E R}(\rho) \leq \frac{1}{\pi} \int_{0}^{\frac{(M-1) \pi}{M}} \\
& \left(1+\frac{C \rho}{\sin ^{2}(\theta)} \beta(\mathbf{X})^{\frac{-1}{M_{T} M_{R}}}\right)^{-M_{T} M_{R}} d \theta
\end{aligned}
$$

It is clear that the diversity order of $M_{T} M_{R}$ is achieved.

\section{OTHER SCENARIOS}

The proposed scheme can also be employed in flat channels presenting time variation and/or spatial correlation. It is also important to evaluate the performance of the proposed technique when only imperfect channel state information (CSI) is available. Hence, in the next sections, we briefly describe the models we adopted for the two aforementioned channel effects, as well as the channel estimation techniques that provide the estimated CSI.

\section{A. Time varying and spatially correlated channel model}

In typical wireless communication systems, transmitters, receivers, and even the scatterers are not static, which may cause channel variation with time. This time variation is usually modeled as a wide-sense stationary stochastic process. Considering the well known Clark/Jakes fading model [18], [20], [21], the channel coefficients are modeled as independent, zero-mean, complex Gaussian random variables with time autocorrelation function

$$
\mathbb{E}\left[h_{(i, j, k)} h_{(i, j, t)}^{*}\right]=\mathcal{J}_{o}\left(2 \pi f_{D} T_{s}|k-t|\right),
$$

where $h_{(i, j, k)}$ models the channel coefficient between the $i$-th transmit $\left(i=1, \ldots, M_{T}\right)$ and $j$-th $\left(j=1, \ldots, M_{R}\right)$ receive antennas at instant $k, \mathcal{J}_{o}$ is the zero-order Bessel function of the first kind, $f_{D} T_{s}$ is the normalized Doppler rate (assumed the same for all of the transmit-receive pairs), $T_{s}$ is the time interval needed to send one space-time codeword and $f_{D}$ is the maximum Doppler shift, computed as $f_{D}=\frac{v \cdot f_{c}}{c}$ for a carrier frequency $f_{c}$, a relative velocity between transmitter and receiver of $v \mathrm{~m} / \mathrm{s}$ and the speed of light $c$.

From the time autocorrelation function (23), the normalized spectrum for each channel coefficient can be expressed as

$$
S(f)=\left\{\begin{array}{cl}
\frac{1}{\pi f_{D}} \frac{1}{\sqrt{1-\left(\frac{f}{f_{D}}\right)^{2}}}, & |f|<f_{D} \\
0, & \text { otherwise. }
\end{array}\right.
$$

It is important to highlight that this power spectrum characterizes isotropic channels, where the transmit antennas radiate uniformly in all directions.

Since the time autocorrelation function (23) is nonrational and its spectrum (24) is bandlimited, it is usual to develop approximations to those expressions for analysis, channel estimation, and simulation purposes. Hence, following [16], [22], [23], we herein approximate the time evolution of channel coefficients by first order auto-regressive, $\operatorname{AR}(1)$, processes due to their simplicity. This is possible since the first 
few correlation terms of (23), for small lags, capture most of the channel dynamics [23]. Therefore, the time-varying nature of each channel coefficient is given by

$$
h_{(i, j, k)}=\beta h_{(i, j, k-1)}+w_{k},
$$

where $\beta=\mathcal{J}_{o}\left(2 \pi f_{D} T_{s}\right)$ and $w_{k}$ is an independent, circularly symmetric, zero-mean, Gaussian excitation noise with variance $\sigma_{w}^{2}=\left(1-\beta^{2}\right)$.

In the previous sections, all the channels in a MIMO system are supposed to be spatially uncorrelated. However, depending on the propagation environment, the polarization of the antenna elements and the spacing between them, spatial correlations may appear between channel coefficients. One of the most simple and used spatially correlated channel models splits the fading correlation into two independent components called receive correlation and transmit correlation, respectively. This channel model, called Kronecker model, can be written as [3]

$$
\mathbf{H}_{k}^{\text {corr }}=\mathbf{R}_{M_{R}}^{1 / 2} \mathbf{H}_{k} \mathbf{R}_{M_{T}}^{1 / 2},
$$

where $\mathbf{H}_{k}^{\text {corr }}$ represents a MIMO channel with spatially correlated coefficients, $\mathbf{R}_{M_{R}}$ and $\mathbf{R}_{M_{T}}$ model the correlation between receive and transmit antennas, respectively, $(\cdot)^{1 / 2}$ stands for the Hermitian square root of a matrix and $\mathbf{H}_{k}$ is a MIMO channel with independent, uncorrelated and unit variance Gaussian elements at time instant $k$.

As the correlated channel model (26) is derived from the channel with uncorrelated coefficients, it is possible to formulate the time evolution of spatially correlated channels in a way analog to (25). Then, by defining $\mathbf{h}_{k}=\operatorname{vec}\left(\mathbf{H}_{k}\right) \in$ $\mathcal{C}^{M_{R} M_{T} \times 1}$ as the vector resulting from stacking the columns of $\mathbf{H}_{k}$ on top of each other, we can express the time-varying AR(1) correlated channel model as [16]

$$
\mathbf{h}_{k}^{\text {corr }}=\beta \mathbf{h}_{k-1}^{\text {corr }}+\mathbf{G w}_{k},
$$

where $\mathbf{h}_{k}^{\text {corr }}$ is a vector with spatially correlated channel coefficients at instant $k, \mathbf{G}=\mathbf{R}_{M_{T}}^{1 / 2} \otimes \mathbf{R}_{M_{R}}^{1 / 2} \in \mathcal{C}^{M_{R} M_{T} \times M_{R} M_{T}}$ and $\mathbf{w}_{k}$ is $\mathcal{C N}\left(0, \sigma_{w}^{2} \mathbf{I}\right)$.

It is worth noting that the model in (27) is quite general. For instance, when $\mathbf{R}_{M_{R}}$ and $\mathbf{R}_{M_{T}}$ are both diagonal matrices, the channels are spatially uncorrelated and (27) reduces to (25). When there is no relative motion between transmitter and receiver, the maximum Doppler shift $f_{D}$ equals to zero and, consequently, $\beta=1$ and $\sigma_{w}^{2}=0$. In this case, (27) reduces to $\mathbf{h}_{k}^{\text {corr }}=\mathbf{h}_{k-1}^{\text {corr }}$, i.e., time-invariant channels such as the ones considered previously in Sections III and IV.

\section{B. Channel estimation}

Most previous works on space-time coding and antenna selection, such as [4], [5], [11], [22], [24], consider that the channel is perfectly known at the receiver. Unfortunately, in practice this channel information is not normally available. Therefore, channel estimation techniques are essential for $\mathrm{MRC}$ receivers to correctly decode the received signals.

In general, channel estimators can be divided into three categories: supervised, unsupervised (or blind) and semiblind algorithms. In supervised channel estimation, a training (known) sequence is periodically sent to the receiver. By comparing the training sequence to the corresponding received signals, the algorithms are able to estimate the channel. Blind algorithms, on the other hand, do not use any training sequence, basing the estimation purely on the received signals and on the statistical properties of the transmitted signals. For this reason, blind algorithms generally demand more computational power than supervised ones. Finally, semiblind techniques combine characteristics of both supervised and unsupervised algorithms, using not just the training sequence, but also the data symbols to estimate the channel. Since most of the communication systems has some sort of training sequence, we focus only on supervised and semiblind channel estimators.

When the channel is quasi-static, as it was assumed in Sections II, III and IV, methods such as those presented in [3], [17] can be successfully used. In this paper, we consider the minimum mean squared error (MMSE) estimator for quasistatic channel scenarios. This estimator is derived in [3], [17] and its final expression is written as

$$
\hat{\mathbf{H}}_{\mathrm{MMSE}}=\mathbf{Y}\left(\mathbf{P}^{H} \mathbf{R}_{H} \mathbf{P}+\sigma_{n}^{2} \mathbf{I}\right)^{-1} \mathbf{P}^{H} \mathbf{R}_{H},
$$

where $\mathbf{P} \in M_{T} \times \tau$ is the matrix with the training sequences sent by all of transmit antennas, $\mathbf{R}_{H} \in M_{T} \times M_{T}$ is the channel correlation matrix and $\sigma_{n}^{2} \mathbf{I}$ is the noise covariance matrix. In cases where the channel is time-varying, the estimation algorithms must be able to track these channel variations, causing the development of adaptive algorithms. One of the most simple and widely known approaches in adaptive filtering theory is the Least Mean Square (LMS) algorithm [25]. The update equations for the $j$-th receive antenna and $k$-th instant are given by

$$
\begin{aligned}
\boldsymbol{\varepsilon}_{j, k} & =\mathbf{y}_{j, k}-\mathbf{P}_{k}^{\mathrm{T}} \mathbf{h}_{j, k}^{*} \\
\mathbf{h}_{j, k+1} & =\mathbf{h}_{j, k}+\mu \mathbf{P}_{k} \varepsilon_{j, k}^{*},
\end{aligned}
$$

where $\mu$ is the step-size, that controls the converge speed and the final estimation error. The LMS algorithm can operate in both training and decision-directed (DD) modes. In the training mode, pilot (known) symbols are available to the receiver, so the matrix $\mathbf{P}_{k}$ is known. Once the transmission of the training sequence is finished, the algorithm enters the DD mode (semiblind), where the matrix $\mathbf{P}_{k}$ is formed by the decisions provided by the MRC decoder. Although this could lead to an error propagation, in this paper we use the simplifying assumption, as in [23], that these decisions are correct.

\section{Vi. Codebook Design for $M_{T}=4 N T$, Simulation RESULTS AND DISCUSSION}

According to new technologies in MIMO systems, which can have much more than four transmit antennas available, we extend the proposed scheme for any number of antennas that is a multiple of four, i,e., $M_{T}=4 N T$ transmit antennas and $M_{R} \geq 1$, where $N T \in \mathbb{Z}^{*}{ }_{+}$. As described before, the 
symbol $s$ should be preprocessed by a complex transmission codeword $\mathbf{x}$ :

$$
\mathbf{x}=\frac{1}{\sqrt{M_{T} / 2}}\left[\begin{array}{c}
\cos (\theta) e^{j \varphi_{0}} \\
\cos (\theta) e^{j \varphi_{l-1}} \\
\vdots \\
\cos (\theta) e^{j \varphi_{l}} \\
\sin (\theta) e^{j \varphi_{0}} \\
\sin (\theta) e^{j \varphi_{l+1}} \\
\vdots \\
\sin (\theta) e^{j \varphi_{L}}
\end{array}\right]
$$

where $l=M_{T} / 2$. The main cost of this extension is the minimum number of feedback bits $b=M_{T}$ required, which is essential to ensure full diversity of BER performance.

From (1) and (31), the received signal $\mathbf{y}$ for $M_{R}$ receive antennas can be rewritten as

$$
\mathbf{y}=\mathbf{H p}+\mathbf{n},
$$

where

$$
\begin{aligned}
\mathbf{H p} & =s \mathbf{H x}=\overline{\mathbf{h}} \\
\overline{\mathbf{h}} & =s\left[h_{p 1} \ldots h_{p M_{R}}\right]^{\mathrm{T}},
\end{aligned}
$$

in which

$$
\begin{aligned}
h_{p j}= & \cos (\theta) h_{1, j}+\cos (\theta) e^{\varphi_{1}} h_{2, j}+\cdots \\
+ & \cos (\theta) e^{\varphi_{l-1}} h_{\left(M_{T}-3\right), j}+\cos (\theta) e^{\varphi_{l}} h_{\left(M_{T}-2\right), j} \\
& +\sin (\theta) h_{3, j}+\sin (\theta) e^{\varphi_{2}} h_{4, j}+\cdots \\
+ & \sin (\theta) e^{\varphi_{L-1}} h_{\left(M_{T}-1\right), j} \sin (\theta) e^{\varphi_{L}} h_{M_{T}, j} .
\end{aligned}
$$

Considering the received signal in (32) and employing the MRC technique, the following processing produces the desired inputs to the maximum-likelihood detector:

$$
\begin{aligned}
& \tilde{s}= \\
& s\left[\left(\sum_{j=1}^{M_{R}}\left(\left|h_{1, j}\right|^{2}+\cdots+\left|h_{l, j}\right|^{2}\right)+\sum_{j=1}^{M_{R}} \beta_{C j}\right) \cos ^{2}(\theta)\right. \\
& \left.+\left(\sum_{j=1}^{M_{R}}\left|h_{l+1, j}\right|^{2}+\cdots+\left|h_{L, j}\right|^{2}\right)+\sum_{j=1}^{M_{R}} \beta_{S j}\right) \sin ^{2}(\theta) \\
& \left.+\sum_{j=1}^{M_{R}} \beta_{C S j} \cos (\theta) \sin (\theta)\right] \sqrt{\rho} / 2+\sum_{j=1}^{M_{R}} H_{p j}^{*} \eta_{j}
\end{aligned}
$$

where

$$
\begin{aligned}
\beta_{C j}= & \sum_{m=1}^{l-1} \sum_{n=1}^{l-1} 2 \Re\left\{g_{m, j} g_{n, j}^{*}\right\}, \\
\beta_{S j}= & \sum_{m=l}^{L} \sum_{n=l}^{L} 2 \Re\left\{g_{m, j} g_{n, j}^{*}\right\}, \quad m \neq n, \\
\beta_{C S j}= & 2 \Re\left\{\left(g_{1, j}+\ldots+g_{l / 2, j}\right)\right. \\
& \left.\times\left(g_{l / 2+1, j}+\ldots+g_{L, j}\right)^{*}\right\}, \\
\mathbf{G}= & \mathbf{H} \odot \mathbf{V}_{\varphi}
\end{aligned}
$$

$$
\mathbf{V}_{\varphi}=\left[\begin{array}{ccc}
e^{j \varphi_{0}} \ldots e^{j \varphi_{l}} & e^{j \varphi_{0}} & e^{j \varphi_{l+1}} \ldots e^{j \varphi_{L}} \\
\vdots & \vdots & \vdots \\
e^{j \varphi_{0}} \ldots e^{j \varphi_{l}} & e^{j \varphi_{0}} & e^{j \varphi_{l+1}} \ldots e^{j \varphi_{L}}
\end{array}\right]
$$

In this section, we present the simulation results used to assess the performance of the proposed scheme. We also compare the obtained BER performance of the proposed scheme with other two closed-loop codebook-based schemes with four transmit antennas: the extended orthogonal spacetime block code scheme (EO-STBC) [9], the Alamouti-code based scheme (ACBS) [11] and the DFT [14]. For the simulations, we assume that the symbols are mapped into a QPSK constellation, the receiver sends $b$ bits over an error-free and zero-delay feedback channel, and the stopping criterion is the occurrence of 300 symbol errors per average SNR. The BER performance for the no-diversity scenario (singleinput single-output scheme) is also plotted in Fig. 1, used as a reference curve. The performance results are compared in terms of bit error rate (BER) versus $\rho$ over quasi-static flat Rayleigh fading channels.

In Figs. 1 (a) and (b), results are given for $M_{T}=4$ transmit antennas and $M_{R}=1$ receive antenna. Fig. 1 shows the BER performance of the proposed, the EO-STBC and the ACBS schemes. For Fig. 1 (a), we assumed that there is an ideal (unquantized) feedback channel between transmitter and receiver in order to assess the best performance that can be reached by the schemes. We can observe that the proposed scheme achieves diversity order of $M_{T} M_{R}=4$ (the same order achieved by the other ones) and has a performance gain of about $3 \mathrm{~dB}(b=\infty)$ and $1.3 \mathrm{~dB}(b=3)$ over the other schemes.

In Fig. 2 we compare the BER performances of the proposed codebooks to that of the uniform DFT codebook presented in [14]. The DFT and the proposed scheme have approximately the same BER performances for $M_{T}=4, M_{R}=1$, and $b=4$ feedback bits. However, as the number of feedback bits is increased, the proposed scheme presents a considerable BER improvement, while the DFT one has just a little performance gain. The codebook presented in [14] offers a good BER performance for a limited quantized feedback channel. A drawback of this solution is the BER performance saturation imposed by the orthogonality of the DFT, which is not observed in our proposal since the codebook design is optimized for quasi-orthogonal codes. Additionally, those solutions have the same design complexity since in both cases the codebooks are based on trigonometrical functions.

Figure 3 (a) presents the theoretical upper bounds for the proposed scheme with $M_{T}=4, M_{R}=1$ and $M_{R}=2$. We have considered the codebooks $\mathbf{X}_{8}^{4}$ for $b=3$ and $\mathbf{X}_{16}^{4}$ for $b=4$ (see Table I and II for more details). We can note that the simulated and theoretical performances get closer and closer as the number of feedback bits is increased because of the approaches we adopted for the derivations. Fig. 2 (b) illustrates the BER performance for an extended version of the proposed scheme. In this case, the scheme has $M_{T}=8$ antennas, for $b=7,8$ and the codes follow the same rule as was described earlier. More details about this extended scheme 


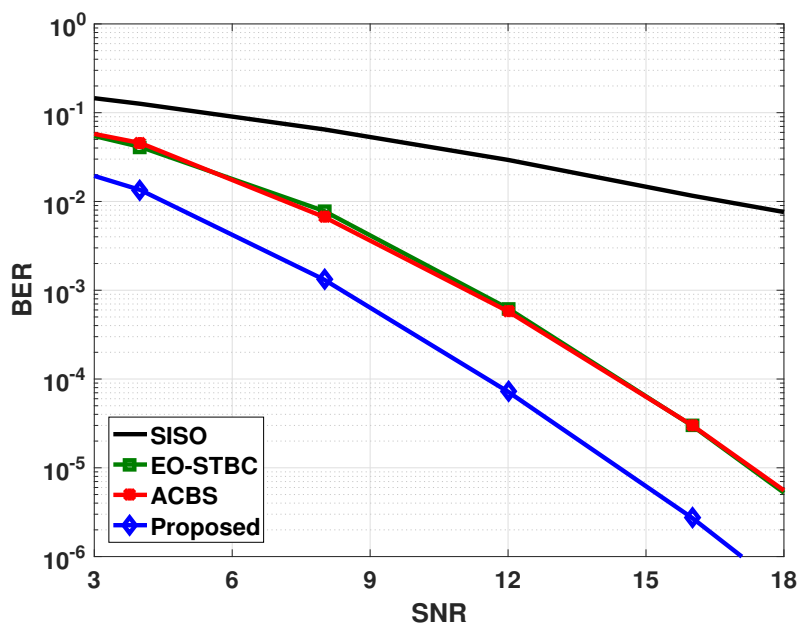

(a)

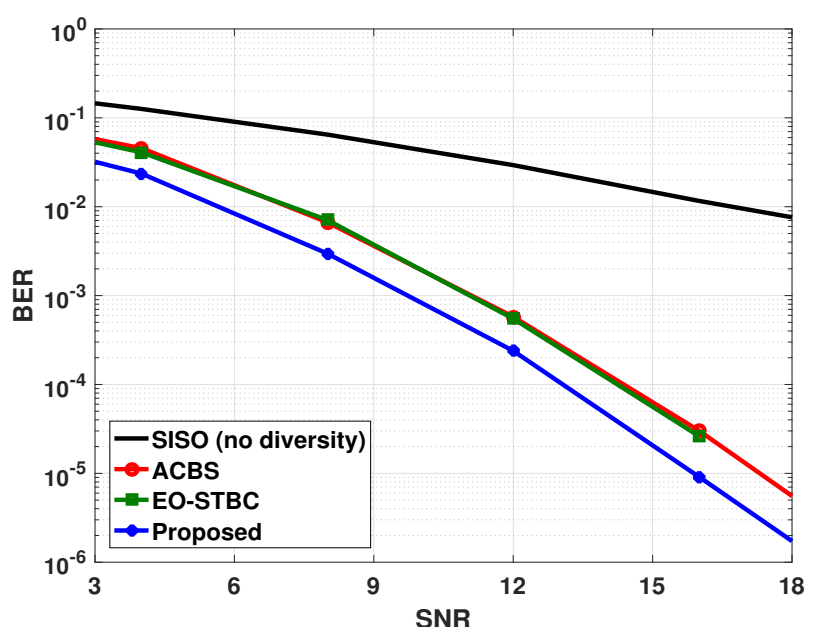

(b)

Fig. 1. BER performances for $M_{T}=4$ and $M_{R}=1$ for the EO-STBC [9], ACBS [11] and the proposed schemes (a) assuming a unquantized feedback channel $(b=\infty)$, (b) assuming a quantized feedback channel with $b=3$.

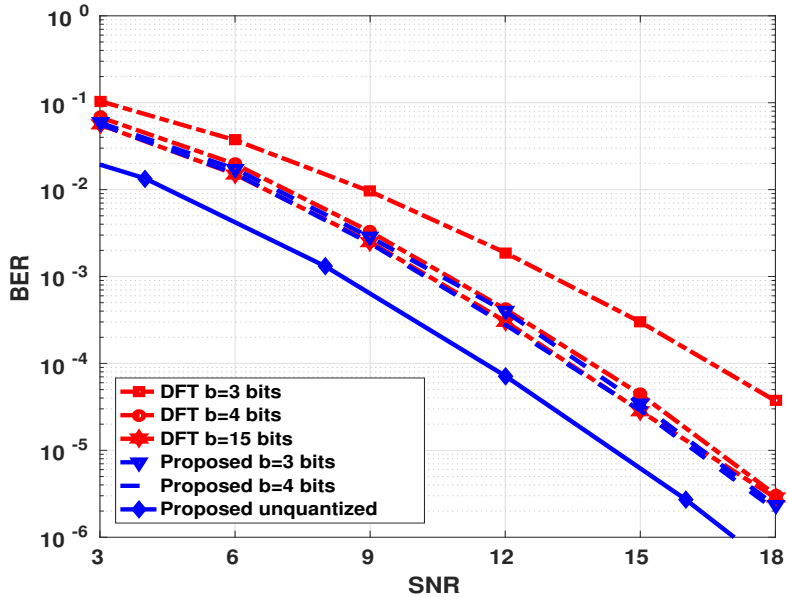

Fig. 2. BER performances for $M_{T}=4$ and $M_{R}=1$ for the DFT [14] and the proposed scheme assuming a quantized feedback channel with $b=3,4$ and unquantized feedback.

will be presented in the next section. As one can see, the diversity order of $8 M_{R}$ is obtained in both cases.

Figures 4 (a) and (b) state the performance of the proposed scheme for a scenario with fast flat fading. The length of the training sequence takes $25 \%$ of the whole transmit frame. For these cases, all of the simulations have used $b=3$. In Fig. 4 (a), we assess the BER performance of the proposed scheme for both the LMS and MMSE estimators at the receiver. We have assumed a relative velocity of $100 \mathrm{~km} / \mathrm{h}$ in both scenarios. As a result, the LMS estimator provided a better performance when compared with the MMSE.

We evaluate the robustness of the proposed scheme by adding spatial correlation in the simulations. Thus, in Figs. 5 (a) and (b) we evaluate the BER performance when the communication is simultaneously affected by the Doppler
TABLE III

BER PERFORMANCE OF THE PROPOSED SCHEME UNDER DOPPLER EFFECT AND SPATIAL CORRELATION. IT WAS ASSUMED $\rho=15 d B$

\begin{tabular}{l|c||c|c}
\hline Doppler & BER & Correlation C & BER \\
\hline \hline $0 \mathrm{~km} / \mathrm{h}$ & $10^{-4.9}$ & 0 & $10^{-4.9}$ \\
\hline $5 \mathrm{~km} / \mathrm{h}$ & $10^{-4.8}$ & 0.2 & $10^{-4.7}$ \\
\hline $50 \mathrm{~km} / \mathrm{h}$ & $10^{-4.4}$ & 0.3 & $10^{-4.7}$ \\
\hline $100 \mathrm{~km} / \mathrm{h}$ & $10^{-3.8}$ & 0.5 & $10^{-4.5}$ \\
\hline $150 \mathrm{~km} / \mathrm{h}$ & $10^{-3.1}$ & 0.8 & $10^{-3.6}$ \\
\hline
\end{tabular}

effect and the spatial correlation. Again, we have assumed a relative velocity of $100 \mathrm{~km} / \mathrm{h}$ and $b=3$. Additionally, in Fig. 5 (b), we have considered two different level of spatial correlation, i.e., $C=0.2$ and $C=0.5$. Based on the results, we can assert that the proposed scheme presents a better BER performance when compared to the ACBS for different channel conditions. More details on these results can be found in Table III.

Note that in this paper we focus our attention in how to explore the spatial diversity available at radio base stations for wireless communications without add much complexity in the system. In terms of real-world scenarios, one can boost this potential by simply dividing a cell into multiple sectors, thus increasing network capacity, such as in multilayer sectorization [26], where the transmit antennas are distributed into the sectors, where the proposed scheme can still be considered as a good solution.

Regarding the results presented in the simulation results, we showed that the proposed scheme keeps a good bit error performance for scenarios with low correlation and low Doppler. The performance degradation presented for either high Doppler or high spatial correlation can be easily explained. For high spatial correlation at the transmitter the orthogonal structure is lost, which is undesirable, since the proposed scheme explores the "quasi-orthogonal" structure of 


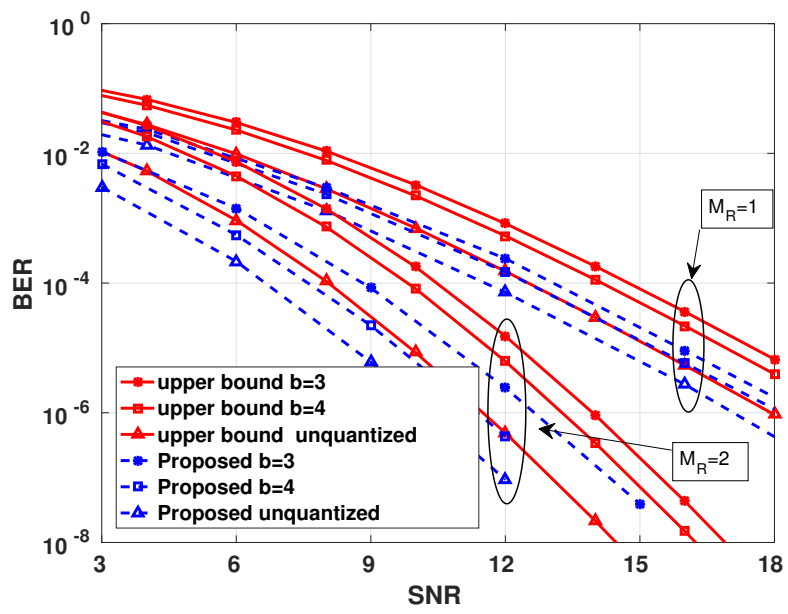

(a)

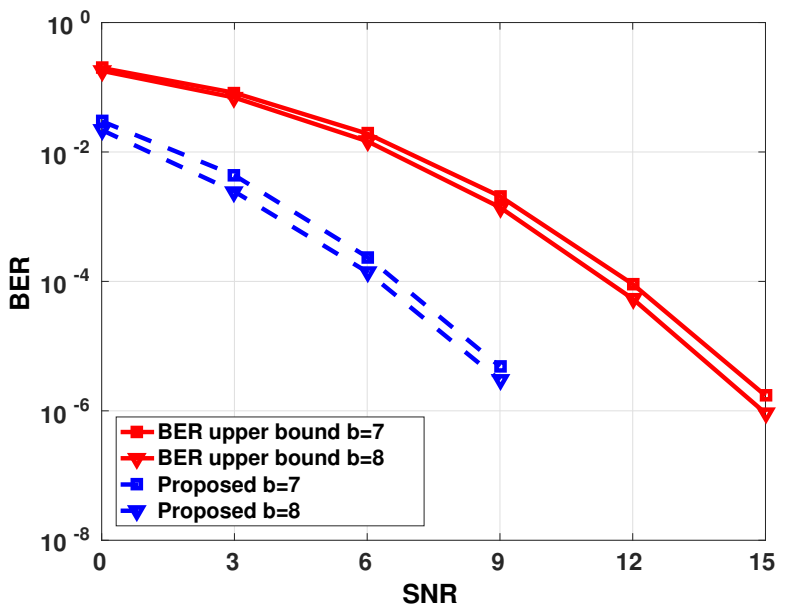

(b)

Fig. 3. BER performances comparing the upper bound to the simulations (a) $M_{T}=4, M_{R}=1$ and $M_{R}=2$ (b) $M_{T}=8, M_{R}=1$ and $b=7,8$.

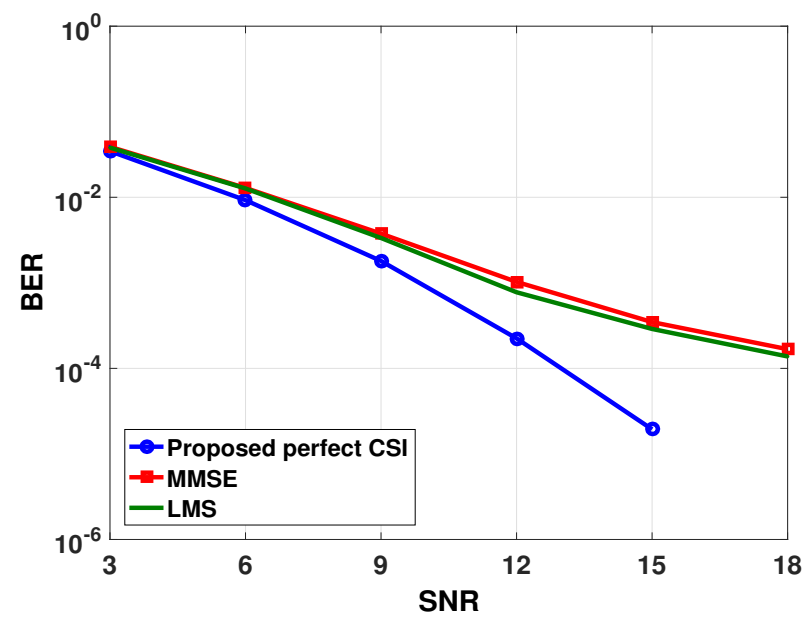

(a)

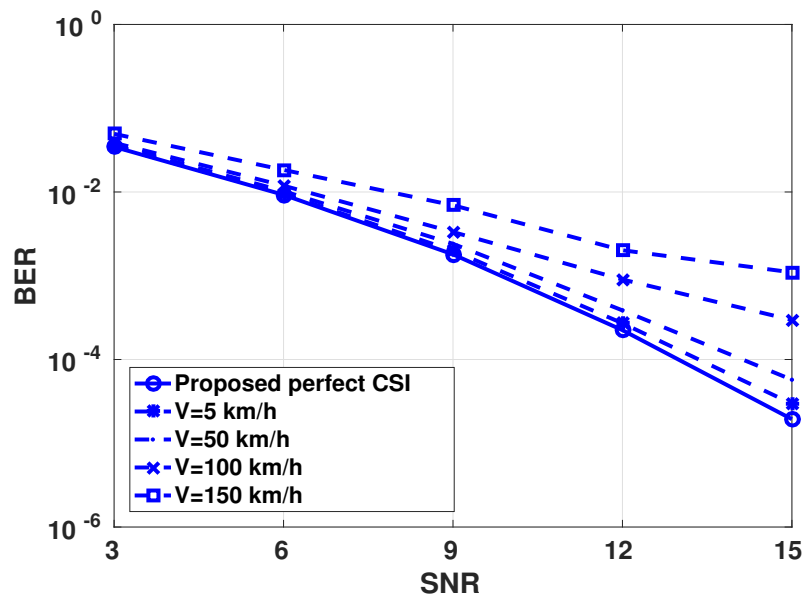

(b)

Fig. 4. Performance comparison of the proposed scheme (a) for the LMS and MMSE channel estimators, (b) the LMS with different relative velocities.

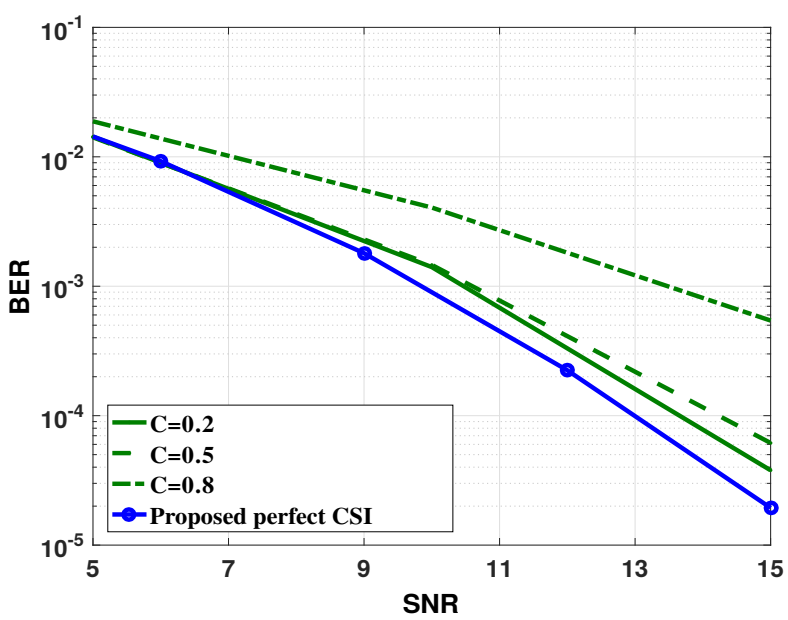

(a)

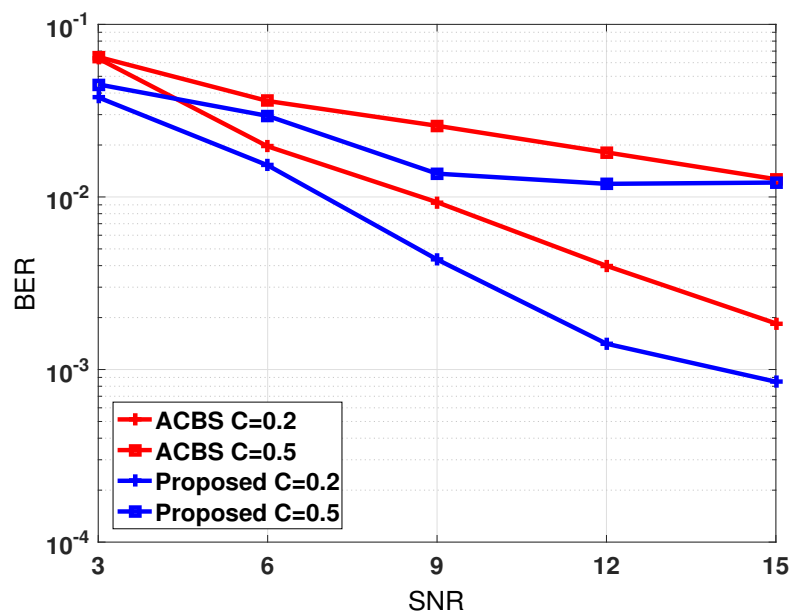

(b)

Fig. 5. BER performance of the proposed scheme by using the LMS estimator (a) while varying the spatial correlation level $C$, (b) as compared to the ACBS scheme[11]. 
the codes. The high mobility makes the channel estimation less accurate, and, as a result, more cross terms will appear in the SNR expression which can not be controlled by the proposed model. For the fast fading scenario the performance of the proposed scheme can be improved by using the estimator based on the Kalman filter [27], for instance.

\section{CONCLUSIONS AND FinAl REMARKS}

A low-complexity codebook-based beamforming with quantized feedback channel was proposed. The proposed scheme is based on a preprocessing that combines the phase rotation and power allocation (suboptimal in this proposal), culminating into a new simple codebook transmission scheme. The proposal can be seen as a code selector scheme, which allows us to evaluate it as a codebook-based design. An instantaneous SNR analysis was addressed in order to find the optimal feedback to be sent to the transmitter. The proposed scheme was evaluated in terms of BER performance through Monte Carlo simulations. Further, a quantized feedback analysis over quasi-static flat Rayleigh fading channels was considered, and an upper bound on the BER performance was derived. The proposed scheme was evaluated for other more realistic scenarios, where we have assumed a spatial correlation at the transmitter and relative mobility between transmitter and receiver. The results showed that the proposed scheme keeps a good bit error performance for scenarios with low correlation and low Doppler. Finally, we have presented how to extend the proposed codebook design to any MIMO system with $M_{T}=4 N$ antennas.

\section{REFERENCES}

[1] M. Skoglund and G. Jongren, "On the capacity of a multipleantenna communication link with channel side information," IEEE Journal on Selected Areas in Communications, vol. 21, no. 3, pp. 395-405, 2003. DOI: 10.1109/JSAC.2003.809696.

[2] P. Xia and G. B. Giannakis, "Design and analysis of transmit-beamforming based on limited-rate feedback," IEEE Transactions on Signal Processing, vol. 54, no. 5, pp. 1853-1863, 2006. DOI: 10.1109/TSP.2006.871967.

[3] E. G. Larsson and P. Stoica, Space-Time Block Coding for Wireless Communications, 1st. New York, NY, USA: Cambridge University Press, 2008.

[4] D. Gore and A. Paulraj, "MIMO antenna subset selection with space-time coding," IEEE Transactions on Signal Processings, vol. 50, no. 10, pp. 2580-2588, 2002. DOI: 10.1109/TSP.2002. 803337.

[5] Z. Chen, J. Yuan, and B. Vucetic, "Analysis of transmit antenna selection/maximal-ratio combining in rayleigh fading channels," IEEE Transactions on Vehicular Technology, vol. 54, no. 4, pp. 1312-1321, 2005. DOI: 10.1109/TVT. 2005.851319.

[6] R. A. Pitaval and O. Tirkkonen, "Joint GrassmannStiefel quantization for MIMO product codebooks," IEEE Transactions on Wireless Communications, vol. 13, no. 1, pp. 210-222, 2014. DOI: 10.1109/TWC.2013.111313.130208.

[7] A. Medra and T. N. Davidson, "Flexible codebook design for limited feedback systems via sequential smooth optimization on the Grassmannian manifold," IEEE Transactions on Signal Processing, vol. 62, no. 5, pp. 1305-1318, 2014. DOI: 10 . 1109/TSP.2014.2301137.
[8] Y. T. Wu, Y. Y. Zhao, and F. Yu, "Comparison of codebooks for beamforming in limited feedback MIMO systems," in 2012 IEEE International Conference on Computer Science and Automation Engineering (CSAE), vol. 2, 2012, pp. 32-36. DOI: 10.1109/CSAE.2012.6272722.

[9] N. M. Eltayeb, S. Lambotharan, and J. A. Chambers, "A phase feedback based extended space-time block code for enhancement of diversity," in 2007 IEEE 65th Vehicular Technology Conference - VTC2007-Spring, 2007, pp. 2296-2299. DOI: 10.1109/VETECS.2007.474.

[10] P. Zhu, L. Tang, Y. Wang, and X. You, "An upper bound on the SER of transmit beamforming in correlated rayleigh fading," IEEE Transactions on Communications, vol. 58, no. 2 , pp. 457-462, 2010. DOI: 10.1109/TCOMM.2010.02.0800442.

[11] I. Choi, J.-K. Kim, H. Lee, and I. Lee, "Alamouti-codes based four-antenna transmission schemes with phase feedback," Communications Letters, vol. 13, no. 10, pp. 749-751, 2009. DOI: 10.1109/LCOMM.2009.090229.

[12] A. Dowhuszko and J. Hämäläinen, "Performance of transmit beamforming codebooks with separate amplitude and phase quantization," IEEE Signal Processing Letters, vol. 22, no. 7, pp. 813-817, 2015. DOI: 10.1109/LSP.2014.2370762.

[13] V. Sharma and S. Sharma, "Novel linear decodable QO-STBC for four transmit antennas with transmit antenna shuffling," Wireless Personal Communications, vol. 82, no. 1, pp. 47-59, 2015. DOI: $10.1007 / \mathrm{s} 11277-014-2192-2$.

[14] J. Suh, C. Kim, W. Sung, J. So, and S. W. Heo, "Construction of a generalized DFT codebook using channel-adaptive parameters," IEEE Communications Letters, vol. 21, no. 1, pp. 196-199, 2017. DOI: 10.1109/LCOMM.2016.2606432.

[15] S. T. Valduga, D. I. Alves, R. Machado, A. P. Legg, and M. B. Loiola, "Low-complexity codebook-based beamforming with four transmit antennas and quantized feedback channel," in 2014 IEEE Wireless Communications and Networking Conference (WCNC), 2014, pp. 1212-1217. DOI: 10.1109/ WCNC.2014.6952322.

[16] M. B. Loiola, R. R. Lopes, and J. M. T. Romano, "Modified kalman filters for channel estimation in orthogonal spacetime coded systems," IEEE Transactions on Signal Processing, vol. 60, no. 1, pp. 533-538, 2012. DOI: 10.1109/TSP.2011. 2170682.

[17] S. M. Kay, Fundamentals of Statistical Signal Processing: Estimation Theory. Upper Saddle River, NJ, USA: PrenticeHall, Inc., 1993.

[18] M. K. Simon and M.-S. Alouini, Digital communication over fading channels, ser. Wiley series in telecommunications and signal processing. Hoboken, N.J. Wiley-Interscience, 2005.

[19] S. Zhou, Z. Wang, and G. B. Giannakis, "Quantifying the power loss when transmit beamforming relies on finite-rate feedback," IEEE Transactions on Wireless Communications, vol. 4, no. 4, pp. 1948-1957, 2005. DOI: 10.1109/TWC.2005. 850259.

[20] M. C. Jeruchim, P. Balaban, and K. S. Shanmugan, Eds., Simulation of Communication Systems: Modeling, Methodology and Techniques, 2nd. Norwell, MA, USA: Kluwer Academic Publishers, 2000.

[21] G. L. Stuber, Principles of Mobile Communication, 1st. Norwell, MA, USA: Kluwer Academic Publishers, 1996.

[22] B. Balakumar, S. Shahbazpanahi, and T. Kirubarajan, "Joint MIMO channel tracking and symbol decoding using Kalman filtering," IEEE Transactions on Signal Processing, vol. 55, no. 12 , pp. 5873-5879, 2007. DOI: 10.1109/TSP.2007.901663.

[23] C. Komninakis, C. Fragouli, A. H. Sayed, and R. D. Wesel, "Adaptive multi-input multi-output fading channel equalization using Kalman estimation," in 2000 IEEE International Conference on Communications. ICC 2000. Global Convergence Through Communications. Conference Record, vol. 3, 2000, 1655-1659 vol.3. DOI: 10.1109/ICC.2000.853775 
[24] Z. Liu, X. Ma, and G. B. Giannakis, "Space-time coding and kalman filtering for time-selective fading channels," IEEE Transactions on Communications, vol. 50, no. 2, pp. 183-186, 2002. DOI: $10.1109 / 26.983312$

[25] S. Haykin, Adaptive Filter Theory (3rd Ed.) Upper Saddle River, NJ, USA: Prentice-Hall, Inc., 1996.

[26] K. Zheng, L. Zhao, J. Mei, B. Shao, W. Xiang, and L. Hanzo, "Survey of large-scale MIMO systems," IEEE Communications Surveys Tutorials, vol. 17, no. 3, pp. 1738-1760, 2015. DOI: 10.1109/COMST.2015.2425294.

[27] E. A. Wan and R. V. D. Merwe, "The unscented Kalman filter," in Kalman Filtering and Neural Networks, 2000, pp. 153-158.

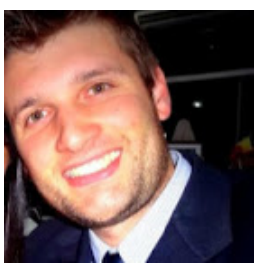

Samuel Tumelero Valduga received the B.S.E.E and M.Sc. degrees in Electrical Engineering and Computer Science from Federal University of Santa Maria (UFSM), Santa Maria, RS, Brazil, in 2012 and 2014, respectively.

$\mathrm{He}$ is currently pursuing his Ph.D. degree at Federal University of Ceará (UFC). He is member of Wireless Telecommunications Research Group (GTEL), Fortaleza, Brazil. From 2016 to 2017, he held a one-year research position at the I3S Laboratory, CNRS, France. His research interests are massive MIMO, matrix and tensor completion, pilot contamination, beamforming, precoding, and channel estimation.

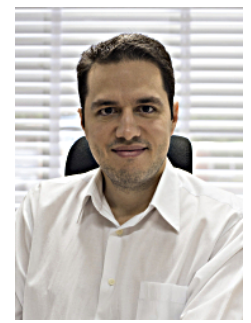

André $\mathrm{L}$. F. de Almeida received the double $\mathrm{Ph}$. degree in Sciences and Teleinformatics Engineering from the University of Nice, Sophia Antipolis, France, and the Federal University of Ceará, Fortaleza, Brazil, in 2007.

$\mathrm{He}$ is currently an Assistant Professor with the Department of Teleinformatics Engineering of the Federal University of Ceará. During fall 2002, he was a visiting researcher at Ericsson Research Labs, Stockholm, Sweden. From 2007 to 2008, he held a one year research position at the I3S Laboratory, CNRS, France. In 2008, he was awarded a CAPES/COFECUB research fellowship with the I3S Laboratory, CNRS, France. He currently holds a productivity research fellowship from CNPq (the Brazilian National Council for Scientific and Technological Development). In 2012, 2013 and 2015, he was a Visiting Professor at the University of Nice Sophia Antipolis, France. He served as an Associate Editor of the IEEE Transactions on Signal Processing (2012-1016). He is currently an Associate Editor of the IEEE Signal Processing Letters and serves as a general co-chair of IEEE CAMSAP2017. His research interests lie in the areas of signal processing for communications and sensor array processing, and include blind methods for channel identification, equalization and source separation, MIMO receivers, multilinear algebra and tensor-based signal processing applied to communications and data analysis.

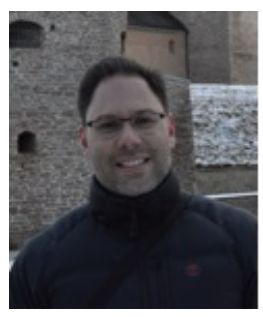

Renato Machado received the B.S. degree in Electrical Engineering from the São Paulo State University, Brazil, in 2001, and the M.Sc. degree and the Ph.D. degree in Electrical Engineering from the Federal University of Santa Catarina (UFSC) Brazil, in 2004 and 2008, respectively.

$\mathrm{He}$ was a visiting Ph.D. researcher in the Department of Electrical Engineering, Arizona State University, from Aug. 2006 to Jun. 2007. He worked as a researcher at Nokia Institute of Technology, in 2007 and 2008. Between Aug. 2009 and Dec. 2017,

he was a professor in the Department of Electronics and Computing, Federa University of Santa Maria, Brazil. From Dec. 2011 to Oct. 2013 he was the director of the Santa Maria Space Science Laboratory. Between Nov. 2013 and Mar. 2015, Dr. Machado spent his sabbatical license as a visiting researcher fellow at Blekinge Institute of Technology, Karlskrona, Sweden, working in partnership with the Saab Electronic Defence Systems group. Dr. Machado is a member of the IEEE Communications Society, IEEE Aerospace and Electronic Systems Society, and the Brazilian Telecommunications and Signal Processing Society (SBrT). Since Dec. 2017, Dr. Machado has been an Associate Professor at the Aeronautics Institute of Technology. His research interests include SAR image processing, wireless communications, MIMO systems, power line communications and sensor networks.

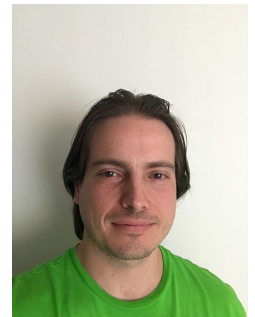

Andrei Piccinini Legg received his B.S. degree in 2005, M.Sc in 2007, and Ph.D in 2011 in Electrical Engineering from the Federal University of Santa Catarina - Brazil.

$\mathrm{He}$ is currently an Associate Professor at the Federal University of Santa Maria - Brazil. His research focus includes image processing, electronic design, error correction codes, and wireless communications.

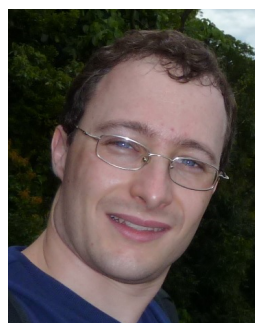

Murilo Bellezoni Loiola received his B.S. in 2002 , M.Sc in 2005, and Ph.D. in 2009 in Electrical Engineering from the University of Campinas (UNICAMP), Brazil.

$\mathrm{He}$ is currently an Assistant Professor at Federal University of $\mathrm{ABC}$ (UFABC). His main research interests lie in the areas of adaptive and statistical signal processing, wireless communications, wireless physical-layer security, and machine learning.

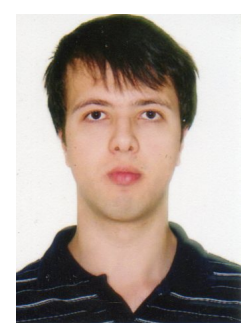

Dimas Irion Alves received the B.S.E.E degree from the Federal University of Santa Maria, Santa Maria, RS, Brazil, in 2013. He received his M.Sc. degree in Electrical Engineering from the Federal University of Santa Catarina, Florianópolis, SC, Brazil, in 2015 .

$\mathrm{He}$ is currently a Ph.D. student at UFSC and a member of the Communications Research Group (GPqCom/UFSC). Since October 2015 he has been a Professor in Federal University of Pampa (Unipampa), Alegrete, RS, Brazil. His research interests include digital signal processing, MIMO systems, wireless communications, SAR systems and image processing. 